\title{
Correlação entre habilidades básicas de leitura e compreensão de leitura
}

\author{
Correlation between basic reading \\ skills and reading comprehension
}

\author{
Vera Lúcia Orlandi CUNHA \\ Cláudia da SILVA \\ Simone Aparecida CAPELLINI'
}

\begin{abstract}
Resumo
Este estudo objetivou verificar a correlação entre habilidades básicas de leitura (tempo, velocidade, precisão) e compreensão de leitura de 80 escolares do $2^{\circ}$ ao $5^{\circ}$ ano do Ensino Público Municipal, divididos em oito grupos, conforme o ano escolar e a presença ou não de dificuldade de aprendizagem. Como procedimento, utilizou-se a leitura oral de textos correspondentes ao ano escolar, seguida de perguntas abertas para verificação da compreensão de leitura. Em todos os grupos, houve diferença estatisticamente significante com correlação entre tempo de leitura e velocidade de leitura. No grupo VI, foi observada correlação entre palavras lidas incorretamente e velocidade de leitura, enquanto o grupo VII apresentou correlação entre palavras lidas incorretamente, velocidade de leitura e compreensão de leitura. Esses dados sugerem que a habilidade de compreensão de leitura requer capacidades cognitivas de alto nível, que vão além de habilidades básicas, sendo fundamental elaborar uma representação mental do conteúdo proposicional para obter o significado global da mensagem escrita.
\end{abstract}

Unitermos: Aprendizagem. Compreensão de leitura. Leitura.

\begin{abstract}
The aim of this study was to verify the correlation between basic reading skills (time, speed, accuracy) and reading comprehension of 80 schoolchildren from $2^{\text {nd }}$ to $5^{\text {th }}$ grade of the Municipal Public Education system, divided into eight groups according to grade (four groups without learning disability and four with learning disability). The procedure used was oral reading of texts related to the school gradefollowed by open questions to check reading comprehension. In all groups there was statistically significant correlation between reading time and speed. In Group VI, correlation was observed between words read incorrectly and reading speed. Whereas group VII presented correlation between words read incorrectly, speed and reading comprehension. These data suggested that reading comprehension skill requires highlevel cognitive capacity that goes beyond basic skills, and it is fundamental for the reader to develop a mental representation of propositional content in order to obtain the overall meaning of the written message.
\end{abstract}

Uniterms: Learning. Reading comprehension. Reading.

$\boldsymbol{\nabla \nabla \nabla \nabla}$

1 Universidade Estadual Paulista Júlio de Mesquita Filho, Faculdade de Filosofia e Ciências, Departamento de Fonoaudiologia. R. Hygino Muzzi Filho, 737, 17525-900, Marília, SP, Brasil. Correspondência para/Correspondence to: V.L.O. CUNHA. E-mail: <verafono@uol.com.br>.

Agradecimentos: À Coordenação de Aperfeiçoamento de Pessoal de Nível Superior e ao Conselho Nacional de Desenvolvimento Científico e Tecnológico pela concessão de bolsa de doutorado para as duas primeiras autoras deste estudo. 
O processo de leitura envolve diversas habilidades cognitivas, como a decodificação de palavras, aquisição de vocabulário, percepção, memória, bem como a compreensão das ideias do texto para a criação de modelos mentais e a compreensão do texto com base no contexto e no ponto de vista do leitor (Johnson, Archibald \& Tenenbaum, 2010; Kintsch, 1998; Kintsch \& Van Dijk, 1978).

A leitura envolve, portanto, dois componentes básicos: o reconhecimento de palavras e as habilidades de compreensão leitora. Essas habilidades de compreensão textual envolvem a visão integrada do texto e a interpretação de sua estrutura, além de processos inferenciais e monitoramento da compreensão. Logo, a compreensão da leitura está na dependência de vários fatores que, juntos, contribuem para que ela seja concretizada. Assim, fatores como eficiência na decodificação, domínio do vocabulário, capacidade de fazer inferências e fatores sociais, bem como a memória operacional, conduzem o leitor ao sentido da mensagem escrita. Dessa forma, apesar de o reconhecimento de palavras e as habilidades de compreensão estarem relacionados, eles são sustentados por habilidades distintas, que predizem a variação no desempenho de tarefas de compreensão, envolvendo a capacidade de integrar as informações do texto, o conhecimento de sua estrutura, o monitoramento metacognitivo e a memória de trabalho (Cunha, Oliveira \& Capellini, 2010; Giangiacomo \& Navas, 2008; Kida, Chiari \& Ávila, 2010; Kintsch, 1998; Kintsch \& Van Dijk, 1978).

Para Morais (1996), existe uma relação entre automatismo (precisão e rapidez) no reconhecimento de palavras e compreensão de leitura. Quanto mais rápida é a identificação de uma palavra, maior a capacidade da memória de trabalho consagrada às operações de análise sintática, de integração semântica dos constituintes da frase e de integração das frases na organização textual, as quais são processos importantes para a compreensão da leitura.

Dessa forma, a compreensão da leitura está na dependência do reconhecimento automático das palavras. Esse reconhecimento foi explicado por Pinheiro (1994) Morais (1996), e Salles e Parente (2002) por meio do modelo de leitura denominado de Dupla Rota, em que a leitura, em um sistema de escrita alfabética, pode 800 ocorrer, pelo menos, de duas maneiras: por meio de um
Processo Visual Direto (Rota Lexical) ou por meio de um processo envolvendo Mediação Fonológica (Rota Fonológica).

Na leitura pela Rota Lexical, as representações de milhares de palavras familiares são armazenadas em um léxico de entrada visual, que é ativado pela apresentação visual de uma palavra. Isso é seguido pela obtenção do significado a partir do sistema semântico (depósito de todo o conhecimento sobre os significados de palavras familiares) para, então, a palavra ser articulada. No processo de identificação de palavras, o uso da Rota Lexical permite acesso mais rápido ao léxico mental por ser um procedimento de acesso direto ao significado a partir da estrutura gráfica.

Já a Rota Fonológica utiliza o processo de conversão grafema-fonema, traduzindo letras ou grupos de letras em fonemas, ou seja, as representações fonêmicas armazenadas ativam as formas fonológicas das palavras que, por sua vez, levam à ativação das representações semânticas e ortográficas correspondentes (Pinheiro, 1994; Morais, 1996). A Rota Fonológica é um procedimento sequencial, ao menos no início do desenvolvimento da leitura, e, portanto, mais lento que a Rota Lexical.

Sendo assim, a compreensão vai depender da capacidade individual de decodificar rapidamente e de reconhecer palavras isoladas de maneira automática e fluente, sendo bastante prejudicada quando o escolar tem muita dificuldade para reconhecer as palavras (Fletcher, Lyons, Fuchs \& Barnes, 2009; Silva \& Capellini; 2010).

Pesquisadores como Ávila, Carvalho e Kida (2009), Breznitz (2006), Kawano, Kida, Carvalho e Ávila (2011), Saine, Lerkanen, Ahonen, Tolvanen e Lytinen (2010) eSnellings, Van Der Leij, Jong e Block (2009) constataram haver relação entre fluência de leitura, decodificação e compreensão, de forma que o processamento lento da palavra interfere na automaticidade da leitura e, consequentemente, na compreensão. Ou seja, a leitura lentificada das palavras consome a memória de trabalho e, portanto, impede que o escolar se dedique à compreensão do texto. Portanto, o leitor que possui fluência tem maior probabilidade de obter melhor desempenho na compreensão do texto.

Por outro lado, porém, podem existir dificuldades na compreensão da leitura, mesmo que não haja 
problemas com a habilidade de decodificação, ou seja, pode haver escolares com comprometimento específico na compreensão da leitura, mas não na decodificação. Um escolar pode saber decodificar uma palavra, mas, se não conseguir extrair seu significado, será afetada a compreensão do texto como um todo. Dessa forma, pode haver escolares com problemas em compreensão, que não conseguem responder às perguntas feitas ao final da leitura, mas que não apresentam dificuldade no nível das palavras e leem com fluência e facilidade (Bishop \& Snowling, 2004; Cunha et al., 2010, Fletcher et al., 2009).

Para Kintsch (1998), Kintsch e Van Dijk (1978) e A.A.A. Santos, Vendramini, Suehiro e L.A.D. Santos (2006), uma compreensão textual bem sucedida exige processos cognitivos de alto nível - como capacidade de realizar inferências, habilidades linguísticas gerais, habilidades de memória e conhecimento de mundo -, que juntos contribuem para a construção de uma representação macroestrutural do texto.

Diante das considerações acima, este estudo teve por objetivo verificar a correlação entre habilidades básicas de leitura (tempo, velocidade e precisão) e compreensão de leitura, em escolares do $2^{\circ}$ ao $5^{\circ}$ ano do ensino fundamental, com e sem dificuldades de aprendizagem.

\section{Método}

Este estudo foi realizado após aprovação do Comitê de Ética em Pesquisa da Faculdade de Filosofia e Ciências da Universidade Estadual Paulista, campus de Marília, sob Protocolo no 1.881/2008.

\section{Participantes}

Após contato prévio com a escola e a autorização da diretora e da coordenadora de uma escola do município de Marília (SP), participaram deste estudo 80 escolares de ambos os sexos, sendo 43 do sexo feminino e 37 do sexo masculino, matriculados entre o $2^{\circ} \mathrm{e}$ $05^{\circ}$ ano do ensino fundamental.

Foram divididos 8 grupos, da seguinte forma:

Os Grupos Gl, GIl, GIII e GIV foram compostos cada qual por 10 escolares sem dificuldade de aprendi- zagem, do $2^{\circ}$ ano ao $5^{\circ}$ ano respectivamente, na faixa etária de 6 anos e 11 meses a 10 anos e 2 meses. Os escolares foram selecionados pelos professores pelo critério de desempenho satisfatório em leitura e escrita, em avaliação de dois bimestres consecutivos. Após, os escolares foram submetidos a avaliação otorrinolaringológica, audiológica e oftalmológica, com resultados dentro dos padrões de normalidade.

De outro lado, os grupos GV, GVI, GVII e GVIII foram compostos cada qual por 10 escolares com dificuldade de aprendizagem, do $2^{\circ}$ ano ao $5^{\circ}$ ano respectivamente, na faixa etária de 7 anos e 11 meses a 13 anos e 5 meses. Os escolares foram selecionados pelos professores, tendo como critério de inclusão o desempenho insatisfatório em sala de aula (desempenho abaixo do esperado em relação a sua classe) em dois bimestres consecutivos.

Como critério de exclusão foi considerada a deficiência sensorial, motora ou cognitiva, e como critério de inclusão foi considerada a ausência de queixa auditiva ou visual descrita no prontuário escolar e a assinatura do Termo de Consentimento Livre e Esclarecido.

\section{Procedimentos}

Para a realização deste estudo utilizou-se como procedimento a leitura oral de um texto correspondente à série de cada grupo estudado. Os textos foram retirados dos livros didáticos utilizados na rede pública municipal da cidade de Marília e selecionados pelos professores da escola onde foi realizada a pesquisa. Esse tipo de procedimento foi escolhido por não haver no Brasil, até o presente momento, nenhum procedimento padronizado e validado para verificação da compreensão de leitura de textos narrativos.

A leitura oral foi gravada para análise posterior e mensuração do tempo e da velocidade de leitura, como também para verificar a exatidão da leitura, por meio da contagem de palavras lidas incorretamente. O tempo de leitura foi cronometrado, e a velocidade de leitura foi calculada multiplicando-se o número de palavras contidas no texto por 60 segundos (um minuto) e o resultado dividido pelo tempo total de leitura em segundos. Após a leitura, os escolares responderam a perguntas abertas para avaliar a compreensão do texto, conforme descrito na literatura (Condemarin \& Blomquist, 1989). 
A leitura do texto e a compreensão de leitura foram realizadas em uma sessão individual, com duração média de 30 minutos.

Os resultados foram analisados estatisticamente por meio do programa Statistical Package for Social Sciences (SPSS) em sua versão 17.0, sendo adotado nível de significância de 5\% (0,050). Os testes utilizados para análise estatística foram o Teste de Mann-Whitney, com o intuito de verificar possíveis diferenças entre as médias dos grupos formados, e o Teste de Análise de Correlação de Spearman, com o intuito de verificar o grau de relação entre as variáveis.

\section{Resultados}

A análise dos resultados deste estudo foi realizada utilizando a estatística não paramétrica, com o objetivo de medir de forma indireta a variabilidade dos desempenhos dos grupos.

A Tabela 1 apresenta a comparação do desempenho em leitura entre os grupos GI vs. GV, Gll vs. GVI,
GIII vs. GIV e GV vs. GVIII, quando foi aplicado o Teste de Mann-Whitney. Em relação à comparação dos grupos Gl e GV, observou-se que ocorreu diferença estatisticamente significativa para Palavras Lidas Incorretamente (PLI) e para Compreensão de Leitura (CL), sendo que o grupo sem dificuldade Gl apresentou, nessas variáveis, desempenho superior em relação ao grupo com dificuldade. Em relação à comparação do desempenho dos grupos Gll e GVI, foi possível observar que houve diferenças estatisticamente significativas em todas as variáveis, indicando desempenho superior para o grupo sem dificuldade Gll. Na comparação do desempenho dos grupos GIII e GVII, ocorreu diferença estatisticamente significativa em todas as variáveis, indicando também desempenho superior do grupo sem dificuldade GIII. Para a comparação entre os grupos GIV e GVIII, houve diferenças estatisticamente significativas para tempo total de leitura, velocidade de leitura e compreensão de leitura, sugerindo que GIV apresentou desempenho superior em todas essas variáveis.

Pode-se observar, na Tabela 1, que os valores do desvio-padrão apresentados pelos grupos compostos

Tabela 1.Descrição das médias, desvio-padrão e p-valor para a comparação entre os grupos Gl e GV, GIl e GVI, GIII e GVII, GIV e GVIII, em todas as variáveis. Marília (SP), 2010.

\begin{tabular}{|c|c|c|c|c|c|}
\hline Grupos & & $T T L$ & PLI & $V L$ & $\mathrm{CL}$ \\
\hline \multirow[t]{2}{*}{ Gl } & Média & 143,30 & 9,80 & 40,64 & 67,50 \\
\hline & $\mathrm{DP}$ & 84,09 & 7,83 & 21,04 & 31,29 \\
\hline \multirow[t]{2}{*}{ GV } & Média & 232,00 & 15,90 & 26,97 & 35,00 \\
\hline & $\mathrm{DP}$ & 106,02 & 6,42 & 13,05 & 26,87 \\
\hline $\mathrm{Gl} \times \mathrm{GV}$ & Valor de $p$ & 0,059 & $0,026^{*}$ & 0,112 & $0,030^{*}$ \\
\hline \multirow[t]{2}{*}{ Gll } & Média & 206,00 & 9,80 & 75,13 & 75,00 \\
\hline & $\mathrm{DP}$ & 83,11 & 5,65 & 26,91 & 31,18 \\
\hline \multirow[t]{2}{*}{ GVI } & Média & 459,30 & 50,50 & 30,50 & 22,50 \\
\hline & $\mathrm{DP}$ & 429,51 & 52,25 & 21,97 & 32,17 \\
\hline GII x GVI & Valor de $p$ & $0,041^{*}$ & $0,002^{*}$ & $0,002^{*}$ & $0,003^{*}$ \\
\hline \multirow[t]{2}{*}{ GIII } & Média & 168,40 & 6,20 & 98,35 & 85,00 \\
\hline & $\mathrm{DP}$ & 46,10 & 3,80 & 21,68 & 26,87 \\
\hline \multirow[t]{2}{*}{ GVII } & Média & 392,70 & 36,20 & 61,68 & 55,00 \\
\hline & DP & 377,40 & 30,56 & 30,07 & 34,96 \\
\hline GIII x GVII & Valor de $p$ & $0,008^{*}$ & $0,000^{*}$ & $0,006^{*}$ & $0,034^{*}$ \\
\hline \multirow[t]{2}{*}{ GIV } & Média & 210,50 & 15,30 & 78,98 & 77,50 \\
\hline & $\mathrm{DP}$ & 57,81 & 8,54 & 21,00 & 24,86 \\
\hline \multirow[t]{2}{*}{ GVIII } & Média & 439,70 & 26,30 & 40,24 & 37,50 \\
\hline & $\mathrm{DP}$ & 195,83 & 22,60 & 16,17 & 21,25 \\
\hline GIV $\times$ GVIII & Valor de $p$ & $0,002^{*}$ & 0,211 & $0,001^{*}$ & $0,003^{*}$ \\
\hline
\end{tabular}

*Valor de $p$ com diferença significante.

802 TTL: Tempo Total de Leitura; PLI: Palavras Lidas Incorretamente; VL: Velocidade de Leitura; CL: Compreensão de Leitura; DP: Desvio-Padrão. 
por escolares com dificuldades de aprendizagem são elevados em palavras lidas incorretamente nos grupos GVI, GVII e GVIII, como também foram demasiados altos em tempo total de leitura para GVI, GVII e GVIII. Isso se deve ao fato de os grupos serem heterogêneos quanto à faixa etária e às dificuldades de leitura, revelando uma variabilidade nas repostas e consequente aumento dos valores de desvio-padrão da amostra.

Quanto à verificação de correlação entre as variáveis de leitura utilizando a Análise de Correlação de Spearman, a Tabela 2 apresenta correlação negativa forte entre velocidade de leitura e tempo total de leitura para Gl; entre velocidade de leitura e tempo total de leitura para Gll; e entre velocidade de leitura e tempo total de leitura para GV. Para GVI, houve correlação negativa moderada entre velocidade de leitura e palavras lidas incorretamente, e correlação negativa fraca entre velocidade de leitura e tempo total de leitura.

Já na Tabela 3 foi possível verificar que ocorreu correlação negativa forte entre velocidade de leitura e tempo total de leitura para os grupos GIII, GIV, GVII e GVIII.

Para o grupo GVII houve correlação negativa moderada-forte entre compreensão de leitura e palavras lidas incorretamente, e correlação negativa moderada entre compreensão de leitura e tempo total de leitura. Ocorreu, ainda para o grupo GVII, correlação positiva moderada-forte entre compreensão de leitura e velocidade de leitura.

\section{Discussão}

Na comparação entre os grupos com e sem dificuldade de aprendizagem observam-se diferenças estatisticamente significativas.

Os dados sugerem que o desempenho torna-se superior nos grupos de maior grau escolar, apontando que, com o aumento da escolaridade, os grupos sem dificuldade de aprendizagem têm desempenho cada vez mais alto, enquanto com os grupos com dificuldade

Tabela 2. Coeficientes de correlação entre os grupos Gl e GV, e entre os grupos GIl e GVI. Marília (SP), 2010.

\begin{tabular}{|c|c|c|c|c|c|}
\hline Grupos & Variáveis & Correlação & $\Pi \mathrm{TL}$ & PLI & $V L$ \\
\hline \multirow[t]{2}{*}{ Gl } & PLI & Coeficiente de Correlação & 0,512 & & \\
\hline & & Valor de $p$ & 0,130 & & \\
\hline \multirow[t]{2}{*}{ GV } & PLI & Coeficiente de Correlação & 0,456 & & \\
\hline & & Valor de $p$ & 0,185 & & \\
\hline \multirow[t]{2}{*}{ Gl } & $V L$ & Coeficiente de Correlação & $-0,879$ & $-0,622$ & \\
\hline & & Valor de $p$ & $0,001^{*}$ & 0,055 & \\
\hline \multirow[t]{2}{*}{ GV } & $V L$ & Coeficiente de Correlação & $-0,879$ & $-0,334$ & \\
\hline & & Valor de $p$ & $0,001^{*}$ & 0,345 & \\
\hline \multirow[t]{2}{*}{ Gl } & $\mathrm{CL}$ & Coeficiente de Correlação & $-0,515$ & $-0,496$ & 0,369 \\
\hline & & Valor de $p$ & 0,128 & 0,145 & 0,294 \\
\hline \multirow[t]{2}{*}{ GV } & $C L$ & Coeficiente de Correlação & $-0,379$ & $-0,564$ & 0,568 \\
\hline & & Valor de $p$ & 0,280 & 0,090 & 0,087 \\
\hline \multirow[t]{2}{*}{ Gll } & PLI & Coeficiente de Correlação & 0,443 & & \\
\hline & & Valor de $p$ & 0,200 & & \\
\hline \multirow[t]{2}{*}{ GVI } & PLI & Coeficiente de Correlação & 0,382 & & \\
\hline & & Valor de $p$ & 0,276 & & \\
\hline \multirow[t]{2}{*}{ Gll } & $V L$ & Coeficiente de Correlação & $-0,997$ & $-0,460$ & \\
\hline & & Valor de $p$ & $0,000^{*}$ & 0,181 & \\
\hline \multirow[t]{2}{*}{ GVI } & $V L$ & Coeficiente de Correlação & $-0,127$ & $-0,661$ & \\
\hline & & Valor de $p$ & $0,042^{*}$ & $0,038^{*}$ & \\
\hline \multirow[t]{2}{*}{ Gll } & $C L$ & Coeficiente de Correlação & 0,180 & $-0,297$ & $-0,137$ \\
\hline & & Valor de $p$ & 0,620 & 0,405 & 0,706 \\
\hline \multirow[t]{2}{*}{ GVI } & $C L$ & Coeficiente de Correlação & $-0,610$ & $-0,514$ & 0,123 \\
\hline & & Valor de $p$ & 0,061 & 0,128 & 0,734 \\
\hline
\end{tabular}


Tabela 3. Coeficientes de correlação entre os grupos GIII e GVII, e entre os grupos GIV e GVIII. Marília (SP), 2010.

\begin{tabular}{|c|c|c|c|c|c|}
\hline Grupos & Variáveis & Correlação & $\Pi \mathrm{TL}$ & PLI & $V L$ \\
\hline \multirow[t]{2}{*}{ GIII } & PLI & Coeficiente de Correlação & $-0,350$ & & \\
\hline & & Valor de $p$ & 0,322 & & \\
\hline \multirow[t]{2}{*}{ GVII } & PLI & Coeficiente de Correlação & 0,445 & & \\
\hline & & Valor de $p$ & 0,197 & & \\
\hline \multirow[t]{2}{*}{ GIII } & $V L$ & Coeficiente de Correlação & $-0,997$ & $-0,358$ & \\
\hline & & Valor de $p$ & $0,000^{*}$ & 0,310 & \\
\hline \multirow[t]{2}{*}{ GVII } & $V L$ & Coeficiente de Correlação & $-0,988$ & $-0,512$ & \\
\hline & & Valor de $p$ & $0,000^{*}$ & 0,130 & \\
\hline \multirow[t]{2}{*}{ GIII } & $\mathrm{CL}$ & Coeficiente de Correlação & 0,128 & $-0,443$ & $-0,127$ \\
\hline & & Valor de $p$ & 0,726 & 0,200 & 0,726 \\
\hline \multirow[t]{2}{*}{ GVII } & $C L$ & Coeficiente de Correlação & $-0,686$ & $-0,727$ & 0,711 \\
\hline & & Valor de $p$ & $0,029^{*}$ & $0,017^{*}$ & $0,021^{*}$ \\
\hline \multirow[t]{2}{*}{ GIV } & PLI & Coeficiente de Correlação & $-0,128$ & & \\
\hline & & Valor de $p$ & 0,725 & & \\
\hline \multirow[t]{2}{*}{ GVIII } & PLI & Coeficiente de Correlação & 0,321 & & \\
\hline & & Valor de $p$ & 0,366 & & \\
\hline \multirow[t]{2}{*}{ GIV } & $V L$ & Coeficiente de Correlação & $-0,988$ & 0,061 & \\
\hline & & Valor de $p$ & $0,000^{*}$ & 0,868 & \\
\hline \multirow[t]{2}{*}{ GVIII } & $V L$ & Coeficiente de Correlação & $-0,960$ & $-0,256$ & \\
\hline & & Valor de $p$ & $0,000^{*}$ & 0,475 & \\
\hline \multirow[t]{2}{*}{ GIV } & $\mathrm{CL}$ & Coeficiente de Correlação & $-0,239$ & $-0,217$ & 0,194 \\
\hline & & Valor de $p$ & 0,506 & 0,547 & 0,591 \\
\hline \multirow[t]{2}{*}{ GVIII } & $\mathrm{CL}$ & Coeficiente de Correlação & 0,178 & 0,384 & $-0,045$ \\
\hline & & Valor de $p$ & 0,622 & 0,274 & 0,901 \\
\hline
\end{tabular}

*Valor de $p$ com diferença significante.

TTL: Tempo Total de Leitura; PLI: Palavras Lidas Incorretamente; VL: Velocidade de Leitura; CL: Compreensão de Leitura.

de aprendizagem ocorre o inverso, ou seja, seu desempenho vai se tornando inferior. Tais dados sugerem que, à medida que aumenta a quantidade e a dificuldade dos textos para leitura em sala de aula, os escolares com dificuldade de aprendizagem vão apresentando seu desempenho cada vez mais defasado em relação àqueles sem dificuldade, pois estes conseguem abstrair, pela leitura dos textos, as informações necessárias para sua aprendizagem (Akyol \& Ulusoy, 2010; Capellini, Butarelli \& Germano, 2010).

Mesmo entre os escolares com dificuldades de aprendizagem essas diferenças foram verificadas, fato observado pelos elevados valores de desvio-padrão apresentados nos resultados. Os valores elevados podem ter sido encontrados também pelo fato de a amostra deste estudo ser pequena. Assim, uma forma de eliminar essa limitação seria aumentar o número de participantes do estudo e distribuí-los por sexo, idade e escolaridade de forma pareada, possibilitando uma análise estatística paramétrica, capaz de medir a propor- ção exata de variabilidade total das habilidades de leitura encontradas.

Na análise de correlação entre as variáveis de leitura foi verificado que ocorreu correlação negativa entre velocidade de leitura e tempo total de leitura em todos os grupos, indicando que, quanto maior a velocidade de leitura, menor foi o tempo de leitura entre os escolares sem dificuldade de aprendizagem, enquanto com os escolares com dificuldade de aprendizagem ocorreu o oposto - ou seja, à medida em que diminuiu a velocidade de leitura, aumentou o tempo de leitura. Esses achados corroboram os argumentos de Breznitz (2006), Kawano et al. (2011) e Snellings et al. (2009) e que sugerem que a fluência em leitura depende de um acesso rápido ao léxico mental, de forma que o processamento lento da palavra interfere na automaticidade da leitura, assim influenciando na velocidade de leitura e, consequentemente, no tempo total de leitura.

Os achados deste estudo apontam uma correlação negativa entre velocidade de leitura e palavras lidas 
incorretamente somente para o grupo GVI, sugerindo que nesse grupo ocorreu um maior número de palavras lidas incorretamente e uma diminuição na velocidade de leitura. Esses dados indicam que, quanto mais precisa for a leitura de palavras, melhor será a fluência na leitura do texto, o que se reflete diretamente na velocidade de leitura, sugerindo uma relação entre precisão e velocidade de leitura (Fletcher et al., 2009; Johnson, Archibald \& Tenenbaum, 2010; Silva \& Capellini, 2010; Sánchez, Garcia, DeSixte, Castellano \& Rosales, 2008; Sánchez, Garcia \& Gonzalez, 2007).

Os resultados encontrados podem ser explicados por meio do modelo de leitura de Dupla Rota. Dessa forma, os escolares sem dificuldade de aprendizagem que obtiveram uma velocidade maior com a diminuição do tempo de leitura podem ter utilizado tanto a rota lexical quanto a fonológica, melhorando também a precisão de leitura. São habilidades próprias dos leitores habilidosos, o mesmo não ocorrendo para os escolares com dificuldade de aprendizagem, que obtiveram tempo de leitura maior, sugerindo o uso da rota fonológica, mais utilizada por leitores pouco habilidosos ou principiantes na aprendizagem de leitura (D'Amico \& Passolunghi, 2009; Silva \& Capellini, 2010; Saine et al., 2010).

Apenas o grupo GVII (3॰ ano, com dificuldade de aprendizagem) apresentou correlação negativa entre compreensão de leitura e tempo total de leitura, e entre palavras lidas incorretamente e compreensão de leitura. Esses dados sugerem que, nesse grupo, ocorreu aumento no tempo de leitura e diminuição na compreensão textual, bem como aumento no número de palavras lidas incorretamente e desempenho inferior na compreensão, sugerindo que a dificuldade no reconhecimento de palavras tenha sido um importante fator na compreensão. Esses dados corroboram o estudo de Salles e Parente (2002), que referiram que as habilidades de reconhecimento de palavras correlacionam-se com a habilidade de compreensão de leitura textual e com o tempo de leitura.

Para o grupo GVII ocorreu também uma correlação com coeficiente positivo moderado-forte entre velocidade de leitura e compreensão de leitura, indicando que a diminuição na velocidade de leitura levaria à diminuição na habilidade de compreensão do material lido.
Esse dado pode sugerir que o uso preferencial da rota fonológica, por leitores com dificuldade de aprendizagem, pode comprometer, de certa forma, a compreensão e a retenção do texto lido. Talvez por ser uma rota mais lenta de acesso ao léxico e, consequentemente, requerer maior carga de memória de trabalho e atenção consciente nesse processo mais básico, o uso dessa estratégia permitiria a interferência de elementos dentro da história, modificando o significado da mesma, enquanto o uso preferencial da rota lexical levaria a uma maior velocidade de leitura textual e, como consequência, a uma melhor compreensão de leitura (Breznitz, 2006; Morais, 1996; Salles \& Parente, 2002, 2004; Snellings et al., 2009).

Os dados do presente estudo, ao apresentarem, apenas no grupo GVII, correlação negativa entre tempo total de leitura e compreensão de leitura e entre palavras lidas incorretamente e compreensão de leitura, sugerem que a compreensão de leitura é uma capacidade que exige a ativação de conhecimentos essenciais que estão profundamente relacionados com o desenvolvimento do vocabulário, habilidades linguísticas, habilidades de memória, capacidade de realizar inferências e experiência de mundo, além de automatismo e precisão na identificação de palavras, como apontado em estudos anteriores (Cunha et al., 2010; Giangiacomo \& Navas, 2008; Kida et al., 2010; Kinsch, 1998; Kinschi \& Van Dijk, 1978; Salles \& Parente, 2002, 2004; Santos, 2009; Uemura \& Hoshiyama, 2010).

Portanto, com base na análise dos resultados deste estudo, foi possível verificar que a compreensão inclui vários processos cognitivos inter-relacionados, como os processos básicos de leitura, ou seja, o reconhecimento e a extração do significado das palavras impressas. No entanto, apesar de serem requisitos necessários, não são suficientes, uma vez que a compreensão de leitura, por ser uma habilidade complexa, necessita também do envolvimento de processos cognitivos de alto nível para ser concretizada.

Dessa forma, de acordo com estudos anteriores (Bishop \& Snowling, 2004; Cunha et al., 2010; Fletcher et al., 2009; Stivanin \& Scheuer, 2007; Uemura \& Hoshiyama, 2010), podem existir dificuldades na compreensão da leitura, apesar da habilidade em reconhecer a palavra escrita. Portanto, é possível que o escolar saiba decodificar uma palavra, porém, quando não acessa sua 
representação semântica, não consegue abstrair o significado das sentenças, fato que afetará a compreensão global do texto. Assim, pode haver escolares que, embora leiam com fluência e facilidade, não conseguem responder às perguntas feitas ao final da leitura de um texto, na medida em que, para leitores mais habilidosos, uma palavra pode ser lida antes de ocorrer o acesso a seu significado.

\section{Considerações Finais}

Os resultados deste estudo permitem concluir que somente o grupo GVII, composto por escolares com dificuldade de aprendizagem, apresentou correlação entre as habilidades básicas de leitura (tempo, velocidade e exatidão) e a habilidade de compreensão de leitura, indicando que apenas para esse grupo as habilidades básicas de leitura foram um importante fator na compreensão.

Esse importante achado leva a refletir que a habilidade de compreensão de leitura exige outras habilidades além daquelas básicas de automatismo e precisão no reconhecimento de palavras. A reflexão é importante de ser compartilhada com profissionais ligados à área de educação - como professores, pedagogos, psicopedagogos, psicólogos e fonoaudiólogos--, os quais devem estar atentos ao fato de que a habilidade de compreensão de leitura, quando trabalhada em sala de aula, deve estar associada ao ensino e exploração de conhecimentos e estratégias que são fundamentais para a elaboração de uma representação mental do conteúdo proposicional da mensagem escrita.

Dessa forma, além das habilidades básicas de leitura, é necessário que aqueles profissionais utilizem estratégias que envolvam processos cognitivos de alto nível - como habilidades linguísticas gerais, habilidades de memória, capacidade de realizar inferências e conhecimento de mundo -, que contribuam para a construção de uma representação macroestrutural do texto, proporcionando ao leitor a extração do significado global do material lido e a obtenção de uma leitura com sentido.

\section{Referências}

Akyol, H., \& Ulusoy, M. (2010). Pre-service teachers' use of 806 reading strategies in their own readings and future classrooms. Teaching and Teacher Education, 26 (4), 878-884.

Ávila, C. R. B., Carvalho, C. A. F., \& Kida, A. S. B. (2009). Parâmetros de fluência e compreensão de leitura. In T. Barbosa, C. C. Rodrigues, C. B. Mello, S. A. Capellini, R. Mousinho \& L. M. Alves. Temas em dislexia (p.103-113). São Paulo: Artes Médicas.

Bishop, D. V. M., \& Snowling, M. J. (2004). Developmental dyslexia and specific language impairment: same or different? Psychological Bulletin, 130 (6), 858-886.

Breznitz, Z. (2006). Fluency in reading: synchronization of process. Mahwah: Lawrence Erlbaum.

Capellini, S. A., Butarelli, A. P. K. J., \& Germano, G. D. (2010). Dificuldades de aprendizagem da escrita em escolares de $1^{a}$ a $4^{a}$ séries do ensino público. Revista Educação em Questão, 37 (23), 146-64.

Condemarin, M., \& Blomquist, M. (1989). Dislexia: manual de leitura corretiva. Porto Alegre: Artes Médicas.

Cunha, V. L. O., Oliveira, A. M., \& Capellini, S. A. (2010). Avaliação e intervenção na compreensão de leitura. In S. A. Capellini, G. D. Germano \& V. L. O. Cunha (Orgs.), Transtornos de aprendizagem e transtornos da atenção (pp.63-76). São José dos Campos: Pulso.

D'Amico, A., \& Passolunghi, M. C. (2009). Naming speed and effortful and automatic inhibition in children with arithmetic learning disabilities. Learning and Individual Differences, 19 (2), 170-180.

Fletcher, J. M., Lyons, G. R., Fuchs, L. S., \& Barnes, M. A. (2009). Transtornos de aprendizagem da identificação à intervenção. Porto Alegre: Artmed.

Giangiacomo, M. C. P. B., \& Navas, A. L. G. P. (2008). A influência da memória operacional das habilidades de compreensão de leitura em escolares de $4^{a}$ série. Revista da Sociedade Brasileira de Fonoaudiologia, 13 (1), 69-74.

Johnson, T. E., Archibald, T. N., \& Tenenbaum, G. (2010). Individual and team annotation effects on students' reading comprehension, critical thinking, and meta-cognitive skills. Computers in Human Behavior, 26 (6), 1496-1507.

Kawano, C. E., Kida, A. S. B., Carvalho, C. A. F., \& Ávila, C. R. B. (2011). Parâmetros de fluência e tipos de erros na leitura de escolares com indicação de dificuldades para ler e escrever. Revista da Sociedade Brasileira de Fonoaudiologia, 16 (1), 9-18.

Kida, A. S. B., Chiari, B. M., \& Ávila, C. R. B. (2010). Escala de leitura: proposta de avaliação das competências leitoras. Revista da Sociedade Brasileira de Fonoaudiologia, 15 (4), 546-553.

Kintsch, W. (1998). Comprehension: a paradigm for cognition. New York: Cambridge University Press.

Kintsch, W., \& Van Dijk, T. A. (1978). Toward a model of text comprehension and production. Psychology Review, 85 (5), 363-394

Morais, J. (1996). A arte deler. São Paulo: Universidade Estadual Paulista.

Pinheiro, A. M. V. (1994). Leitura e escrita: uma abordagem cognitiva. Campinas: Editora Psy II. 
Saine, N. L., Lerkanen, M., Ahonen, T., Tolvanen, A., \& Lytinen, H. (2010). Predicting word-level reading fluency outcomes in three contrastive groups: remedial and computer-assisted remedial reading intervention, and mainstream instruction. Learning and Individual Differences, 20 (5), 402-414.

Salles, J. F., \& Parente, M. A. M. P. (2002). Processos cognitivos na leitura de palavras em crianças: relações com compreensão e tempo de leitura. Psicologia: Reflexão e Crítica, 15 (2), 321-331.

Salles, J. F., \& Parente, M. A. M. P. (2004). Compreensão textual em alunos da segunda e terceira séries: uma abordagem cognitiva. Estudos de Psicologia (Natal), 9 (1), 71-80.

Sánchez, E., Garcia, J. R., \& Gonzalez, A. J. (2007). Can differences in the ability to recognize words cease to have an effect under certain reading conditions? Journal of Learning Disabilities, 40 (4), 290-305.

Sánchez, E., Garcia, J. R., DeSixte, R., Castellano, N., \& Rosales, J. (2008). El análisis de la práctica educativa y las propuestas instruccionales: integración y enriquecimiento mutuo. Infancia y Aprendizaje, 2 (1), 233-258.

Santos, A. A. A., Vendramini, C. M. M., Suehiro, A, C. B., \& Santos, L. A. D. (2006). Leitura compreensiva e utilização de estratégias de aprendizagem em alunos de Psicologia. Estudos de Psicologia (Campinas), 23 (1), 82-91. doi: 10.15 90/S0103-166X2006000100010.
Santos, M. T. M. (2009). Dislexia: princípios para a intervenção fonoaudiológica. In T. Barbosa, C. C. Rodrigues, C. B. Mello, S. A. Capellini, R. Mousinho \& L. M. Alves. Temas em dislexia. São Paulo: Artes Médicas.

Silva, C., \& Capellini, S. A. (2010). Eficácia do programa de remediação fonológica e leitura no distúrbio de aprendizagem. Pró-Fono Revista de Atualização Científica, 22 (2), 131-138.

Snellings, P., Van Der Leij, A., Jong, P. F., \& Block, H. (2009). Enhancing the reading fluency and comprehension of children with reading disabilities in an orthographically transparent language. Journal of Learning Disabilities, 42 (2), 291-305.

Stivanin, L., \& Scheuer, C. I. (2007). Tempo de latência para a leitura: influência da freqüência da palavra escrita e da escolarização. Revista da Sociedade Brasileira de Fonoaudiologia, 12 (3), 206-13.

Uemura, J., \& Hoshiyama, M. (2010). Effect of task-irrelevant high-speed verbal stimulation on a visual/verbal worddiscrimination task: an event-related potential study. Clinical Neurophysiology, 121 (12), 2065-2069. doi: 10.1016/ j.clinph.2010.05.007.

Recebido em: 7/1/2011

Versão final em: 2/5/2012

Aprovado em: 20/5/2012 
